\title{
Antihyperlipidemic Medication Treatment Patterns and Statin Adherence Among Patients with ASCVD in a Managed Care Plan After Release of the 2013 ACC/AHA Guideline on the Treatment of Blood Cholesterol
}

\author{
Brandon K. Bellows, PharmD, MS; Cody J. Olsen, PharmD, BCPS; \\ Jennifer Voelker, PharmD Candidate; and Curtis Wander, PharmD, BCPS
}

\section{ABSTRACT}

BACKGROUND: The American College of Cardiology (ACC) and American Heart Association (AHA) released a new blood cholesterol treatment guideline in November 2013. It is unknown how the new recommendations have affected cholesterol medication use and adherence in a commercial health plan.

OBJECTIVE: To evaluate the effect of the 2013 guideline release on antihyperlipidemic treatment patterns and statin adherence in patients with atherosclerotic cardiovascular disease (ASCVD) compared with a historical control group.

METHODS: This study was a historical cohort analysis of adult patients (aged 21-75 years) with clinical ASCVD enrolled in a SelectHealth commercial health plan. Patients were included in the guideline implementation cohort if they had a medical claim with an ICD-9-CM diagnosis of ASCVD in the year before the November 2013 ACC/AHA guideline release. The index date was defined as the first outpatient medical claim with an ICD9-CM for ASCVD in the first 6 months after the guideline was released. Patients were required to have continuous enrollment for $\geq 1$ year before and after the index date. These same criteria were applied to patients exactly 4 years earlier to identify a historical control group. Patients meeting these criteria formed the antihyperlipidemic treatment patterns cohort. Of these, patients who also had $\geq 1$ pharmacy claim for a statin in the 1-year pre- and post-index periods were included in the statin adherence cohort. Antihyperlipidemic treatment patterns were assessed using pharmacy claims for antihyperlipidemic medications in the 1-year pre- and post-index periods. Antihyperlipidemic medication claims were classified as a nonstatin cholesterol medication, low-intensity statin, moderateintensity statin, or high-intensity statin. To address differences in pre-index antihyperlipidemic medications between the guideline implementation and historical control groups, patients were randomly matched 1:1 based on pre-index classification in a post hoc analysis. Post-index antihyperlipidemic classifications were compared between groups using a StuartMaxwell test. The change in mean statin adherence (proportion of days covered $[\mathrm{PDC}]$ ) was compared within and between groups using paired and independent t-tests, respectively. The proportion of adherent patients $(P D C \geq 0.80)$ in the pre- and post-index periods was compared between groups using a chi-square test. A multivariable logistic regression was used to compare the likelihood of being adherent in the post-index period while controlling for pre-index adherence and other potential confounders.

RESULTS: A total of 7,818 adult members with ASCVD in the index period and 1 year before the index period were identified. of those, 1,841 patients met the criteria to be included in the analysis, and 1,526 patients were matched on antihyperlipidemic classification and included in the antihyperlipidemic treatment patterns analysis. Baseline characteristics were similar, although the guideline implementation group was younger (58.3 vs. 60.5 years, $P<0.001)$, and more were male $(74.8 \%$ vs. $71.3 \%$, $P=0.106$ ) than the historical control group. In the matched cohort, there was a significant difference in the post-index antihyperlipidemic classification $(P<0.001)$, which appeared to be a result of the difference in nonstatin cholesterol medications (guideline $6.9 \%$ vs. historical $13.0 \%$ ) and high-intensity statins (guideline $23.7 \%$ vs. historical $16.3 \%$ ). Of the 1,841 patients in the antihyperlipidemic treatment patterns cohort, 919 patients met inclusion criteria for the statin adherence analysis. Although PDC decreased over time in both groups, significantly more patients in the guideline implementation group were adherent in the post-index period than the historical control group ( $66.5 \%$ vs. $57.3 \%$, respectively; $P=0.005)$. Additionally, patients in the guideline implementation group were more likely than the historical control to be adherent in the post-index period when adjusting for potential confounders $(\mathrm{OR}=1.49,95 \% \mathrm{Cl}=1.10-2.03$; $P=0.011$ ).

CONCLUSIONS: Since the release of the updated ACC/AHA treatment guideline, more commercial health plan patients with ASCVD used high-intensity statins and fewer used nonstatin cholesterol medications than historical controls. Additionally, since the guideline release, more patients are adherent to statin therapy than historical controls. This study provides managed care organizations with valuable information regarding the effect of the 2013 ACC/AHA guideline.

J Manag Care Spec Pharm. 2016;22(8):892-900

Copyright $\odot 2016$, Academy of Managed Care Pharmacy. All rights reserved.

\section{What is already known about this subject}

The updated 2013 American College of Cardiology (ACC) and American Heart Association (AHA) blood cholesterol treatment guideline represents a major shift in treating atherosclerotic cardiovascular disease (ASCVD).

The 2013 guideline recommendations were projected to increase the number of persons using statin therapy, but real-world observations of how treatment use has changed has been limited. Adherence to statin medications has been shown to be relatively low, and the effect of removing the treat to target cholesterol goals has not been studied. 


\section{What this study adds}

This study compared, among patients with ASCVD, the effect of the ACC/AHA cholesterol guideline on antihyperlipidemic treatment patterns and adherence to a historical control group.

More patients with ASCVD received high-intensity statins and fewer received nonstatin cholesterol medications after the guideline release than historical controls.

A greater proportion of patients with ASCVD were adherent to statin therapy after the guideline release than historical controls.

I n November 2013, the American College of Cardiology (ACC) and the American Heart Association (AHA) released the 2013 ACC/AHA Guideline on the Treatment of Blood Cholesterol to Reduce Atherosclerotic Cardiovascular Risk in Adults, which updated lipid treatment and replaced the longstanding method of treating to blood cholesterol targets established by the Adult Treatment Panel III (ATP III) guideline., Rather than recommending specific low-density lipoprotein cholesterol (LDL-C) goals, the guideline endorses treatment with certain "statin intensities" based on patient characteristics and risk factors. ${ }^{1}$ The new guideline identifies 4 major statin benefit groups, 1 of which is patients with clinical atherosclerotic cardiovascular disease (ASCVD) taking statins for secondary prevention. For patients aged $>75$ years with clinical ASCVD, a moderate-intensity statin is recommended; for adults aged $\leq 75$ years with clinical ASCVD, a high-intensity statin is recommended. ${ }^{1}$

Because of the 2013 guideline recommendations, a projected 12.8 million additional U.S. adults may be eligible for statin therapy. While the majority of this increase will likely occur among adults using statins for the primary prevention of cardiovascular events, ${ }^{3}$ the number of patients eligible for statin therapy as secondary prevention may also increase. However, because of the similar eligibility criteria outlined in the ATP III guideline, this increase will not likely be as drastic., ${ }^{2,3}$ While observational studies are beginning to examine changes to antihyperlipidemic treatment patterns after the release of the 2013 ACC/AHA guideline, they have thus far been limited to a single health system with a relatively small sample size. ${ }^{4}$ The impact of the 2013 ACC/AHA guideline on broader populations remains unknown.

The 2013 ACC/AHA guideline emphasizes adherence to lifestyle modifications and statins as essential components of primary and secondary prevention of ASCVD. ${ }^{1}$ Adherence to statin therapies is critical to reducing the risk of cardiovascular events, and statin nonadherence is a major challenge to optimal management. ${ }^{5-7}$ A meta-analysis of 20 studies revealed that adherence to statins is poor; only $57 \%$ of patients using statins for primary prevention and $76 \%$ using them for secondary prevention are considered adherent. ${ }^{8}$ Furthermore, adherence to statins decreases over time, with as few as $26 \%$ of patients considered adherent after 5 years. ${ }^{9}$ Nonadherent patients receive little or no benefit in reduction of cardiovascular events or mortality., ${ }^{40-13}$ By eliminating the emphasis of LDL-C treatment targets during follow-up, the treatment process may be simplified, but removing LDL-C targets may also affect positive reinforcement of patient adherence behaviors. Overall, the effect that the 2013 ACC/AHA guideline may have had on statin adherence remains unknown.

The purpose of this study was to examine the effect of the 2013 ACC/AHA guideline release on antihyperlipidemic treatment patterns and statin adherence in adult members of a regional managed care organization using a historical control group.

\section{Methods}

\section{Patients and Data Source}

This study was a historical cohort analysis of adult patients with clinical ASCVD enrolled in a SelectHealth commercial health plan. SelectHealth is a nonprofit regional managed care organization that covers approximately 800,000 lives from the Intermountain region, with commercial, Medicare, and Medicaid plans available. Additionally, SelectHealth is part of an integrated health system with Intermountain Healthcare, which is a nonprofit health system with 22 hospitals and over 185 clinics across Utah and Idaho. Data for the study were collected from SelectHealth pharmacy and medical claims. The Intermountain Healthcare Institutional Review Board reviewed and approved this study.

Commercially insured patients were included in the study if they were aged $\geq 21$ years, which corresponds to the age recommendations in the 2013 ACC/AHA cholesterol guideline. ${ }^{1}$ Patients were also required to have a medical claim with an International Classification of Diseases, Ninth Revision, Clinical Modification (ICD-9-CM) diagnosis of ASCVD (Appendix $\mathrm{A}$, available in online article) in the year before the guideline release in the guideline implementation group (i.e., November 12, 2012-November 12, 2013) and over a similar time frame but 4 years before in the historical control group (i.e., November 12, 2008-November 12, 2009). The historical control group was chosen as 4 years before the guideline group, since most patients are enrolled in a health plan for 2-3 years, and a relatively recent comparator would minimize some differences in generic availability of antihyperlipidemic treatments. Because antihyperlipidemic treatment may change for a variety of reasons and drug adherence may change over time, including a comparator group was an important component of this analysis and was used as an effort to isolate the effect of the guideline release. For the guideline implementation group, the index period was November 12, 2013-May 12, 2014, which was the 6-month period following the guideline release, and for 


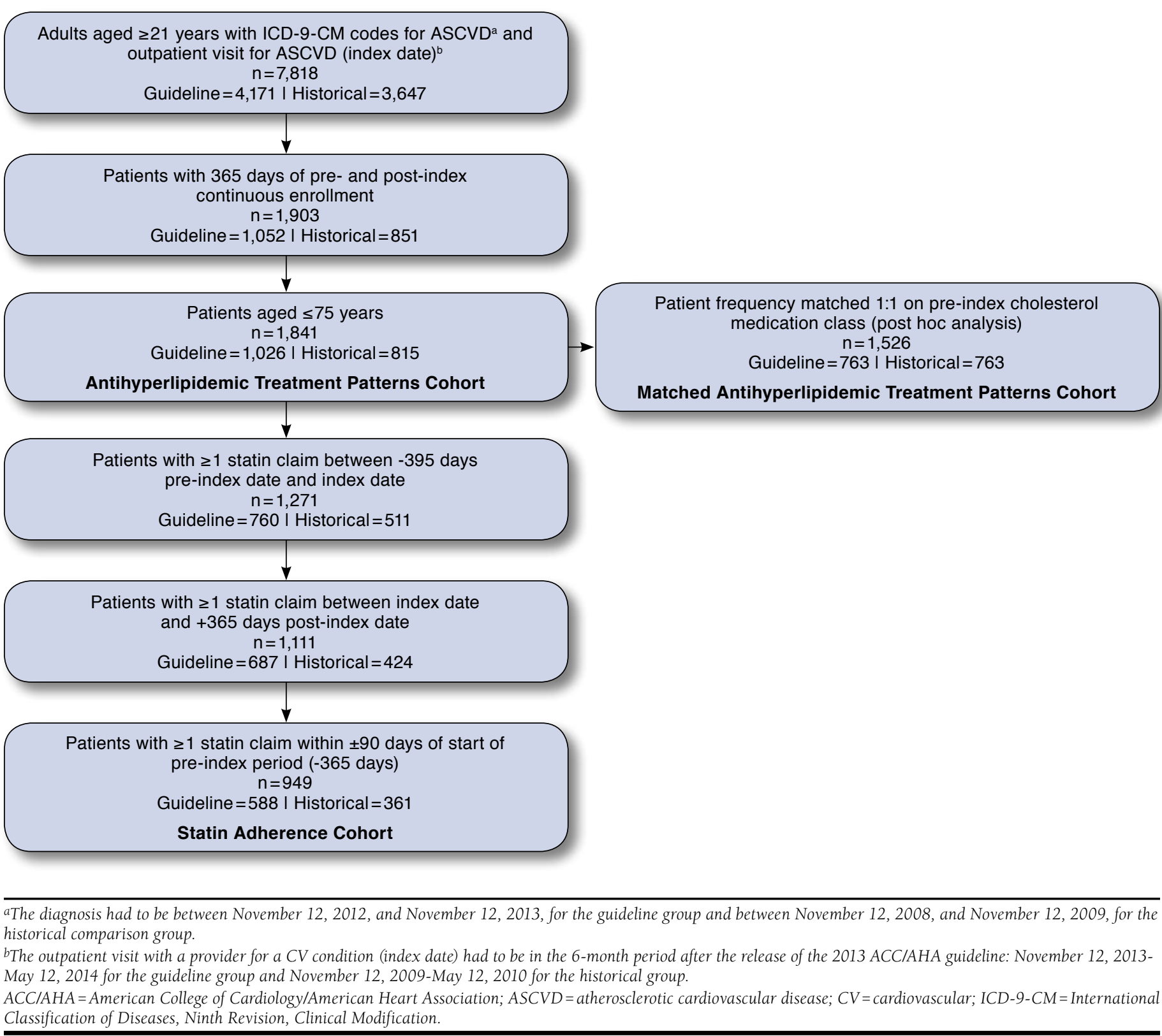

the historical control group, the index period was November 12, 2009-May 12, 2010. The index date was defined as the date of the first cardiovascular- or lipid-related outpatient medical claim during the index period (Appendix A contains a list of ICD-9-CM codes used; ICD-9-CM codes could be in any position). This date was chosen because it represented an opportunity for the provider and patient to discuss and change antihyperlipidemic medications. To ensure that all patients would have the same recommended treatment according to the guideline, patients were also required to be aged $\leq 75$ years on the index date. Additionally, patients were required to have $\geq 365$ days of continuous enrollment in the pre- and post-index periods. Patients meeting these criteria comprised the antihyperlipidemic treatment patterns cohort.

To examine statin adherence, in addition to the previously listed criteria, patients had to have $\geq 1$ pharmacy claim for a statin between -395 days pre-index date and index date as well as $\geq 1$ pharmacy claim for a statin between index date and 


\section{Antihyperlipidemic Medication Treatment Patterns and Statin Adherence Among Patients with ASCVD in a Managed Care Plan After Release of the 2013 ACC/AHA Guideline on the Treatment of Blood Cholesterol}

\section{TABLE 1 Baseline Characteristics of Patients in Antihyperlipidemic Treatment Patterns Cohort $(\mathrm{N}=1,841)$}

\begin{tabular}{|c|c|c|c|c|c|c|c|}
\hline & \multicolumn{2}{|c|}{ Overall $(\mathrm{N}=1,841)$} & \multicolumn{2}{|c|}{ Guideline $(n=1,026)$} & \multicolumn{2}{|c|}{ Historical $(\mathrm{n}=815)$} & \multirow[b]{2}{*}{$P$ Value } \\
\hline & $\mathrm{n}$ & $\%$ & $\mathbf{n}$ & $\%$ & $\mathrm{n}$ & $\%$ & \\
\hline \multicolumn{8}{|l|}{ Demographics } \\
\hline Age, mean [SD] & 59.3 & {$[8.0]$} & 58.3 & {$[7.4]$} & 60.5 & [8.6] & $<0.001$ \\
\hline Male & 1,348 & 73.2 & 767 & 74.8 & 581 & 71.3 & 0.106 \\
\hline \multicolumn{8}{|l|}{ Clinical characteristics } \\
\hline Hyperlipidemia & 1,519 & 82.5 & 862 & 84.0 & 657 & 80.6 & 0.065 \\
\hline Hypertension & 1,366 & 74.2 & 765 & 74.6 & 601 & 73.7 & 0.730 \\
\hline Diabetes & 315 & 17.1 & 172 & 16.8 & 143 & 17.5 & 0.704 \\
\hline Overweight & 16 & 0.9 & 10 & 1.0 & 6 & 0.7 & 0.624 \\
\hline Obesity & 228 & 12.4 & 145 & 14.1 & 83 & 10.2 & 0.013 \\
\hline Anxiety & 22 & 1.2 & 11 & 1.1 & 11 & 1.3 & 0.668 \\
\hline Depression & 49 & 2.7 & 26 & 2.5 & 23 & 2.8 & 0.814 \\
\hline Bipolar & 8 & 0.4 & 1 & 0.1 & 7 & 0.9 & 0.025 \\
\hline Psychotic disorder & 3 & 0.2 & 2 & 0.2 & 1 & 0.1 & 1.000 \\
\hline \multicolumn{8}{|l|}{ Pre-index antihyperlipidemic medication use } \\
\hline \multicolumn{8}{|l|}{ Antihyperlipidemic medication classes } \\
\hline None & 388 & 21.1 & 203 & 19.8 & 185 & 22.7 & \multirow{5}{*}{$<0.001$} \\
\hline Nonstatin medication & 212 & 11.5 & 80 & 7.8 & 132 & 16.2 & \\
\hline Low-intensity statin & 25 & 1.4 & 15 & 1.5 & 10 & 1.2 & \\
\hline Moderate-intensity statin & 742 & 40.3 & 378 & 36.8 & 364 & 44.7 & \\
\hline High-intensity statin & 474 & 25.7 & 350 & 34.1 & 124 & 15.2 & \\
\hline Antihyperlipidemic copay, mean [SD $]^{\mathrm{b}}$ & $\$ 22$ & [\$27] & $\$ 20$ & {$[\$ 27]$} & $\$ 24$ & [\$25] & $<0.001$ \\
\hline Number of antihyperlipidemic fills, mean [SD]b & 7.8 & {$[5.5]$} & 7.3 & {$[4.9]$} & 8.4 & {$[6.1]$} & $<0.001$ \\
\hline \multicolumn{8}{|c|}{$\begin{array}{l}\text { aContinuous variables compared between guideline and historical groups using t-tests, categorical variables compared using chi-square test, or Fisher's exact test } \\
\text { as appropriate. } \\
\text { bAmong patients with at least } 1 \text { antihyperlipidemic fill. } \\
\text { SD=standard deviation. }\end{array}$} \\
\hline
\end{tabular}

+365 days post-index date. Finally, to ensure that patients had similar possible exposures to statins in the pre- and post-index periods, patients were required to have $\geq 1$ pharmacy claim for a statin within \pm 90 days of start of pre-index period ( -365 days \pm 90 days). Patients meeting all of these criteria comprised the statin adherence cohort.

\section{Antihyperlipidemic Treatment Patterns}

Prescription claims for antihyperlipidemic medications were identified using Generic Product Identifier codes in the 1-year pre- and post-index periods. During the pre- and post-index periods, each claim for an antihyperlipidemic medication was classified as a nonstatin cholesterol medication, lowintensity statin, moderate-intensity statin, or high-intensity statin according to the 2013 ACC/AHA guideline definition. ${ }^{1}$ If patients did not have any prescription claims for an antihyperlipidemic medication, they were classified as having none. Patients were then assigned a classification in the preand post-index years according to the highest intensity statin received during the period. If patients received a nonstatin cholesterol medication and a statin, they were classified according to the statin intensity received.

\section{Statin Adherence}

Statin adherence in the pre- and post-index periods was calculated using the proportion of days covered (PDC). PDC was calculated by dividing the number of days supplied by 365 days, taking into account early refills by shifting the start date of the prescription filled early to the end of the days supply of the previous prescription fill and only considering the 365 days during the 1-year pre- and post-index periods. Statin adherence was estimated at the class level, which allowed patients to switch between statins. Fill dates were adjusted if switches were filled early. Additionally, prescription claims from before the start of the pre-index period with a days supply that carried over into the pre-index period were included. If this occurred, only the days actually within the 365-day pre-index period were included in calculating PDC (e.g., a prescription claim with a 30-day supply filled 15 days before the start of the preindex period resulted in 15 days of coverage in the pre-index period). Pharmacy claims from the pre-index period with a days supply that carried over into the post-index period were handled similarly. Patients were considered adherent if they had a PDC $\geq 0.80$ and nonadherent if the PDC was $<0.80$. 

Treatment Patterns Cohort Matched on Pre-index Classification in a Post Hoc Analysis $(\mathrm{N}=1,526)$

\begin{tabular}{|c|c|c|c|c|c|c|c|}
\hline & \multicolumn{2}{|c|}{ Overall $(\mathrm{N}=1,526)$} & \multicolumn{2}{|c|}{ Guideline $(n=763)$} & \multicolumn{2}{|c|}{ Historical $(n=763)$} & \multirow[b]{2}{*}{$P$ Value $^{\mathrm{a}}$} \\
\hline & $\mathbf{n}$ & $\%$ & $\mathbf{n}$ & $\%$ & $\mathrm{n}$ & $\%$ & \\
\hline \multicolumn{8}{|c|}{ Antihyperlipidemic medication classification } \\
\hline None & 386 & 25.3 & 193 & 25.3 & 193 & 25.3 & \multirow{5}{*}{$<0.001$} \\
\hline Nonstatin medication & 152 & 10.0 & 53 & 6.9 & 99 & 13.0 & \\
\hline Low-intensity statin & 24 & 1.6 & 14 & 1.8 & 10 & 1.3 & \\
\hline Moderate-intensity statin & 659 & 43.2 & 322 & 42.2 & 337 & 44.2 & \\
\hline High-intensity statin & 305 & 20.0 & 181 & 23.7 & 124 & 16.3 & \\
\hline
\end{tabular}

aPost-index medication classifications compared for matched pairs using Stuart-Maxwell test.

\section{Statistical Analysis}

Baseline characteristics were identified in the 1-year pre-index period and included demographics (i.e., age and sex) and clinical characteristics (i.e., comorbidities identified by ICD9-CM code included hyperlipidemia, hypertension, diabetes, overweight, obesity, anxiety, depression, bipolar, and psychotic disorders [Appendix A]) as well as average antihyperlipidemic or statin copay. Based on the authors' clinical judgment, comorbidities associated with an increased risk of cardiovascular events or that may affect medication adherence were selected. Continuous variables were described using means and standard deviations (SD) and compared between groups using independent t-tests. Categorical variables were reported using frequencies and percentages and were compared between groups using chi-square or Fisher's exact tests as appropriate. The baseline characteristics of the antihyperlipidemic treatment patterns cohort were described, as well as the subset of patients meeting the criteria of the statin adherence cohort.

Antihyperlipidemic treatment patterns were described using frequencies and percentages and were compared using chi-square tests. However, given the significant differences in antihyperlipidemic classification identified between the groups in the pre-index period, a post hoc analysis was used to better understand and describe changes in treatment patterns. In the post hoc analysis, the post-index antihyperlipidemic classifications were compared between groups after patients in the guideline implementation group were randomly matched 1:1 to patients in the historical control group on pre-index antihyperlipidemic classification. Patients who did not match on pre-index antihyperlipidemic class were excluded from the post hoc analysis. In the post hoc analysis, baseline characteristics were compared between the groups using paired t-tests and McNemar's test or Stuart-Maxwell test, as appropriate, and antihyperlipidemic treatments in the post-index period were compared using the Stuart-Maxwell test.

For the statin adherence cohort, the change in mean statin adherence from the pre- to the post-index periods was compared within the guideline implementation and historical control groups using paired t-tests and between groups using an independent t-test. The proportion of patients considered adherent in the pre- and post-index periods was compared between the guideline implementation and historical control groups using a chi-square test. A multivariable logistic regression was then used to compare the likelihood of being adherent in the post-index period between the guideline implementation and historical control cohorts, while controlling for baseline adherence classification and other potential confounders (i.e., the baseline characteristics previously described). A backward stepwise approach was used in covariate selection for the multivariable logistic regression using a $P$ value of $>0.2$ as a threshold. ${ }^{14}$ Covariates considered clinically meaningful were included regardless of the $P$ value obtained in the stepwise selection process. The final covariates included in the model were age, sex, statin adherence in the pre-index period, mean statin copay in pre-index period, hypertension, anxiety, and depression. All statistical analyses were performed using R version 3.1 (R Foundation for Statistical Computing, Vienna, Austria).

\section{Results}

A total of 7,818 adult patients were identified with an outpatient medical claim for ASCVD in the 6-month index period period, as well as a claim with a diagnosis of ASCVD in the 1 year before (Figure 1). Of those, 1,841 patients met the criteria to be included in the antihyperlipidemic treatment patterns cohort, and 1,526 patients were matched 1:1 on pre-index antihyperlipidemic classification. Of the 1,841 patients in the antihyperlipidemic treatment patterns cohort, 949 patients met all inclusion criteria for the statin adherence cohort.

\section{Antihyperlipidemic Treatment Patterns}

A total of 1,026 patients in the guideline implementation and 815 in the historical control groups were included in the antihyperlipidemic treatment cohort (Table 1). The guideline implementation group was younger (mean [SD] for age: guideline 58.3 [7.4] vs. historical 60.5 [8.6], $P<0.001$ ), had a higher proportion with a diagnosis for obesity (guideline 14.1\% vs. historical 10.2\%, $P=0.013$ ), and had a lower proportion 


\section{Antihyperlipidemic Medication Treatment Patterns and Statin Adherence Among Patients with ASCVD in a Managed Care Plan After Release of the 2013 ACC/AHA Guideline on the Treatment of Blood Cholesterol}

TABLE 3

Baseline Characteristics of Patients in Statin Adherence Cohort ( $N=949)$

\begin{tabular}{|c|c|c|c|c|c|c|c|}
\hline & \multicolumn{2}{|c|}{ Overall $(\mathrm{N}=949)$} & \multicolumn{2}{|c|}{ Guideline $(\mathrm{n}=588)$} & \multicolumn{2}{|c|}{ Historical $(n=361)$} & \multirow[b]{2}{*}{$P$ Value } \\
\hline & $\mathrm{n}$ & $\%$ & $\mathbf{n}$ & $\%$ & $\mathrm{n}$ & $\%$ & \\
\hline \multicolumn{8}{|l|}{ Demographics } \\
\hline Age, mean [SD] & 59.8 & {$[7.0]$} & 58.4 & {$[6.9]$} & 62.2 & {$[6.6]$} & $<0.001$ \\
\hline Male & 742 & 78.2 & 467 & 79.4 & 275 & 76.2 & 0.274 \\
\hline \multicolumn{8}{|l|}{ Clinical characteristics } \\
\hline Hyperlipidemia & 851 & 89.7 & 534 & 90.8 & 317 & 87.8 & 0.172 \\
\hline Hypertension & 727 & 76.6 & 454 & 77.2 & 273 & 75.6 & 0.630 \\
\hline Diabetes & 186 & 19.6 & 120 & 20.4 & 66 & 18.3 & 0.474 \\
\hline Overweight & 8 & 0.8 & 5 & 0.9 & 3 & 0.8 & 1.000 \\
\hline Obesity & 118 & 12.4 & 76 & 12.9 & 42 & 11.6 & 0.613 \\
\hline Anxiety & 8 & 0.8 & 3 & 0.5 & 5 & 1.4 & 0.165 \\
\hline Depression & 26 & 2.7 & 17 & 2.9 & 9 & 2.5 & 0.873 \\
\hline Bipolar & 2 & 0.2 & 1 & 0.2 & 1 & 0.3 & 1.000 \\
\hline Psychotic disorder & 2 & 0.2 & 1 & 0.2 & 1 & 0.3 & 1.000 \\
\hline \multicolumn{8}{|l|}{ Pre-index statin intensity } \\
\hline Low-intensity statin & 17 & 1.8 & 11 & 1.9 & 6 & 1.7 & \multirow{3}{*}{$<0.001$} \\
\hline Moderate-intensity statin & 553 & 58.3 & 296 & 50.3 & 257 & 71.2 & \\
\hline High-intensity statin & 379 & 39.9 & 281 & 47.8 & 98 & 27.1 & \\
\hline Pre-index statin copay, mean [SD] & $\$ 20.02$ & [\$25.19] & $\$ 17.80$ & {$[\$ 24.51]$} & $\$ 23.25$ & {$[\$ 25.81]$} & $<0.001$ \\
\hline \multicolumn{8}{|c|}{$\begin{array}{l}\text { aContinuous variables compared between guideline and historical groups using t-tests, categorical variables compared using chi-square test, or Fisher's exact tests } \\
\text { as appropriate. }\end{array}$} \\
\hline
\end{tabular}

with a diagnosis for bipolar disorder (guideline $0.1 \%$ vs. historical $0.9 \%, P=0.025$ ). Among those patients with $\geq 1$ antihyperlipidemic fill, patients in the guideline implementation group had a lower mean antihyperlipidemic copay (guideline $\$ 20$ vs. historical $\$ 24, P<0.001$ ) and fewer mean antihyperlipidemic fills (guideline 7.3 [4.9] vs. historical 8.4 [6.1], $P<0.001$ ). The characteristics for the matched groups in the post hoc analysis were similar to those before matching on pre-index antihyperlipidemic class, although the difference in bipolar diagnosis was no longer significant $(P=0.077$; Appendix $B$, available in online article).

In the overall treatment patterns cohort before matching, the pre-index medication use classifications were as follows: $21.1 \%$ no cholesterol medication, $11.5 \%$ nonstatin cholesterol medication, $1.4 \%$ low-intensity statin, $40.3 \%$ moderate-intensity statin, and $25.7 \%$ high-intensity statin. Before matching, there was a significant difference in the pre-index antihyperlipidemic classification between the guideline implementation and historical control groups $(P<0.001$; Table 1$)$. This difference was because of the nonstatin cholesterol medications (guideline $7.8 \%$ vs. historical 16.2\%), moderate-intensity statins (guideline $36.8 \%$ vs. historical $44.7 \%$ ), and highintensity statins (guideline $34.1 \%$ vs. historical 15.2\%). After matching on pre-index antihyperlipidemic class, there were no differences in pre-index antihyperlipidemic classifications (both groups: none $24.2 \%$, nonstatin cholesterol medication $10.5 \%$, low-intensity statin $1.3 \%$, moderate-intensity statin
47.7\%, and high-intensity statin 16.3\%; Appendix B). In the matched post hoc cohort, there was a significant difference in the post-index antihyperlipidemic classification between the guideline implementation and historical control groups $(P<0.001 ;$ Table 2). This difference appeared to be a result of a lower use of nonstatin cholesterol medications (guideline 6.9\% vs. historical 13.0\%) and an increased use of high-intensity statins (guideline $23.7 \%$ vs. historical 16.3\%) in the guideline implementation group.

\section{Statin Adherence}

A total of 588 patients in the guideline implementation and 361 in the historical control groups met all the inclusion criteria to be included in the analysis (Table 3). The guideline implementation group was significantly younger than the historical control group (mean [SD]) for age: guideline 58.4 [6.9] vs. historical 62.2 [6.6], $P<0.001$ ). Additionally, there was a significant difference in the statin intensity classification in the pre-index period between the groups $(P<0.001 ;$ Table 3$)$. There were fewer moderate-intensity (guideline $50.3 \%$ vs. historical $71.2 \%$ ) and more high-intensity statin users in the guideline implementation group (guideline $47.8 \%$ vs. historical 27.1\%). The guideline implementation group also had a lower mean [SD] statin copay (guideline \$18 [\$25] vs. historical \$23 [\$26], $P<0.001$ ).

In the overall statin adherence cohort, the mean [SD] PDC in the pre-index period was 0.82 [0.22]; mean PDC was 0.83 [0.20] in the guideline implementation and 0.80 [0.23] in the historical control groups. Adherence decreased from the 


\section{Antihyperlipidemic Medication Treatment Patterns and Statin Adherence Among Patients with ASCVD in a Managed Care Plan After Release of the 2013 ACC/AHA Guideline on the Treatment of Blood Cholesterol}

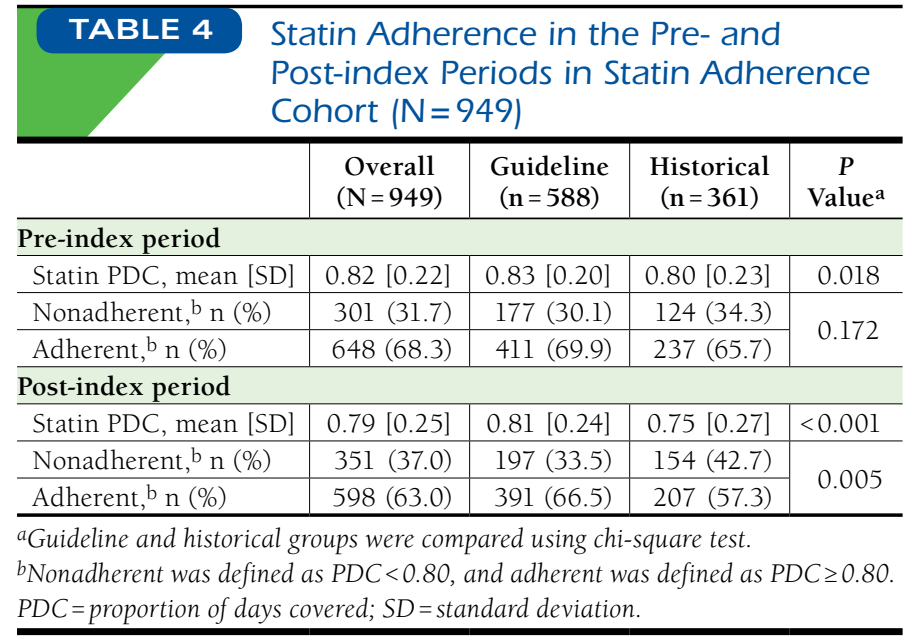

pre- to the post-index periods for each group. The mean ([SD], $P$ value for change) in the post-index period was $0.79(0.25$, $P<0.001)$ for the overall group, $0.81(0.24, P=0.040)$ for the guideline implementation group, and $0.75(0.27, P<0.001)$ for the historical control group. The proportion of patients with a $P D C \geq 0.80$ in the pre-index period was not significantly different between the groups (guideline $69.9 \%$ vs. historical $65.7 \%, P=0.172$; Table 4). However, significantly more patients in the guideline group were considered adherent in the postindex period (guideline $66.5 \%$ vs. historical $57.3 \%, P=0.005$ ). When controlling for potential confounders, patients in the guideline implementation group were significantly more likely than the historical control group (odds ratio $[\mathrm{OR}]=1.49,95 \%$ confidence interval $[\mathrm{CI}]=1.10-2.03, P=0.011$ ) to be adherent in the post-index period (Table 5). However, being considered adherent in the pre-index period was the strongest predictor of being adherent in the post-index period ( $\mathrm{OR}=5.65,95 \%$ $\mathrm{CI}=4.18-7.68, P<0.001)$

\section{Discussion}

This study examined the effect of the 2013 ACC/AHA guideline on antihyperlipidemic treatment patterns and statin adherence in patients with clinical ASCVD in a regional managed care organization. When compared with a historical control group on similar antihyperlipidemic medication classes, patients in the guideline implementation group were more likely to receive high-intensity statins and less likely to receive nonstatin cholesterol-lowering medications in the post-index period. However, according to the 2013 ACC/AHA guideline, the entire antihyperlipidemic treatment patterns cohort should have been taking high-intensity statins. After the release of the guideline, only $37.2 \%$ of the unmatched and $23.7 \%$ of the matched guideline implementation group were on high-intensity statins. Additionally, $22.1 \%$ of the unmatched and $25.3 \%$ of the matched guideline implementation group were untreated.

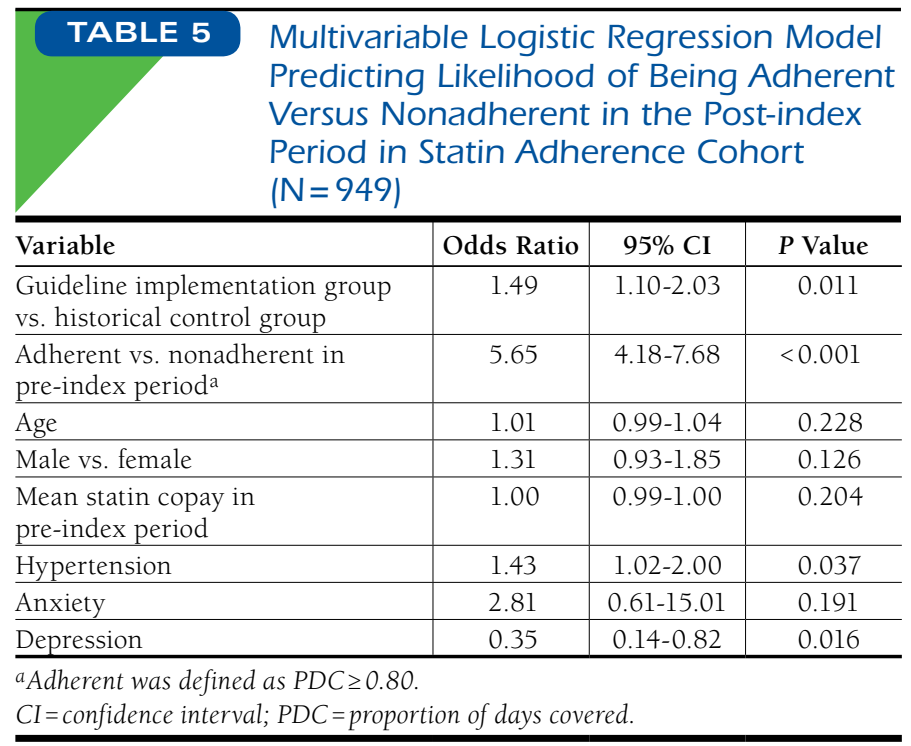

This indicates that, while treatment patterns have shifted since the 2013 ACC/AHA guideline release, treatment inertia may exist, and there is room for improvement. While the entire population of patients should have been on statins according to the ACC/AHA guidelines, some patients may have experienced statin intolerance. In real-world settings, statin intolerance has been estimated to occur in 10\%-15\% of patients treated with statins, so it is likely not all of the untreated patients had statin intolerance..$^{15,16}$ Additionally, patients in the guideline implementation group were more likely than historical controls to be adherent in the post-index period. These results may indicate that, at least initially, the release of the 2013 ACC/AHA guideline affected patient adherence. However, only $67 \%$ of the guideline implementation group had a PDC $\geq 0.80$ in the post-index period, and efforts may have been made to further promote statin adherence.

Although the release of the 2013 ACC/AHA guideline was expected to broadly affect antihyperlipidemic medication use, before this study, the actual effect on treatment patterns and statin adherence was unknown. Because the implementation of new guidelines can take an extensive amount of time, it is important for managed care organizations to assess how patients and providers adapt to new guidelines. These assessments provide opportunities for managed care to encourage good clinical practice among providers and meet quality metrics that change to meet new standards. This study provides managed care organizations with valuable information regarding the effect of the 2013 ACC/AHA guideline and highlights the need for managed care organizations and health systems to consider implementing care process models, provider education, or other interventions to help improve adherence to evidence-based guidelines. 


\section{Antihyperlipidemic Medication Treatment Patterns and Statin Adherence Among Patients with ASCVD in a Managed Care Plan After Release of the 2013 ACC/AHA Guideline on the Treatment of Blood Cholesterol}

The antihyperlipidemic treatment pattern analysis results are similar in direction to a previously published study that examined prescribing patterns in patients with ASCVD seen in primary care clinics in a university health system. ${ }^{4}$ That study found that high-intensity statin use in patients aged between 18 and 75 years had increased from $26 \%$ to $45 \%(P=0.01)$. While the magnitude of effect seen was greater than in the current study, there were several key differences. First, the previous study only included patients seen in primary care clinics from a single university health system and had a relatively small sample size. These parameters may limit the generalizability of the results. Additionally, the previous study used medical records and examined statins prescribed, not necessarily filled. Further, it only examined statin prescription orders for patients during 1 period of time, either before or after the release of the 2013 ACC/AHA guideline. Patients with orders in both periods were randomly assigned to one of the periods. Thus, it did not necessarily consider changes to therapy for patients. The current study overcomes some of these limitations by using claims data from a regional managed care organization, including a historical cohort for comparison, and looking specifically at changes to antihyperlipidemic therapy.

\section{Limitations}

Several limitations of this study are worthy of discussion. First, the index date was assumed to be a point at which the provider and patient had the opportunity to discuss adjusting cholesterol medications and address statin adherence. This was a surrogate marker for the provider's implementation of the new cholesterol guidelines. This surrogate does not identify actual uptake of the guideline in clinical practice, and future research should consider examining this. Historically, guideline adoption has been slow, but information dissemination has changed significantly, and it is unknown how quickly guidelines are adopted. Also, since only claims data were used for this analysis, patients who were not candidates for statin therapy, for reasons such as previous intolerance or ineffectiveness, could not be identified. Future studies may consider using claims and medical record data to overcome this.

Second, the current study only considered patients with ASCVD and did not examine other groups to which statins offer benefits identified in the guideline. Similarly, SelectHealth launched Medicare and managed Medicaid plans in 2013, so these populations were not included in the analysis because of the lack of available data during the study period. Along these lines, Medicare star ratings include a statin adherence measure, and it is unknown how this might have impacted the focus on statin adherence in the overall health plan. While this analysis did not include Medicare patients, the addition of a Medicare plan between the historical group and guideline cohorts may have provided an incidental focus on statin adherence. Future studies should include the effect of the ACC/AHA guideline on Medicare and Medicaid populations.
Finally, patients were only required to have a single ASCVD diagnosis in the pre-index period. Some patients in the antihyperlipidemic treatment cohort may have thus been relatively newly diagnosed and not yet started treatment. Also, by requiring at least 1 claim for a statin in the pre- and post-index periods for the statin adherence cohort, included patients had received statin therapy for some time. This may limit the generalizability of the results of the adherence analysis.

\section{Conclusions}

Since the release of the updated ACC/AHA treatment guideline, more patients with ASCVD are using high-intensity statins, and fewer are using nonstatin cholesterol medications than historical controls. The significant difference between the types of antihyperlipidemic medication use could be a result of prescribing based on the guideline and/or practicing of evidence-based medicine. Additionally, since the guideline release, more patients were adherent to statin therapy than historical controls. The difference in statin adherence could be a result of provider emphasis on adherence, as recommended by the 2013 ACC/AHA guideline, but previous statin adherence was still the largest predictor of adherence. However, the effect of a possible emphasis on improving statin adherence was not studied and should be examined in future studies.

\section{Authors}

BRANDON K. BELLOWS, PharmD, MS, SelectHealth, Murray, Utah, and University of Utah, College of Pharmacy, Salt Lake City, Utah. CODY J. OLSEN, PharmD, BCPS, and CURTIS WANDER, PharmD, BCPS, SelectHealth, Murray, Utah. JENNIFER VOELKER, PharmD Candidate, University of Utah, College of Pharmacy, Salt Lake City, and University of North Carolina, Eshelman School of Pharmacy, Chapel Hill.

AUTHOR CORRESPONDENCE: Brandon K. Bellows, PharmD, MS, SelectHealth, 5381 Green St., Murray, UT 84123. Tel.: 801.442.7790; Fax: 801.442.6870; E-mail: brandon.bellows@selecthealth.org.

\section{DISCLOSURES}

No outside funding or services were received for this work. Outside of the current study, Bellows has received research funding from Biogen Idec, Regeneron Pharmaceuticals, Myriad Genetic Laboratories, Shire Development, and Bristol-Myers Squibb and an honorariam from Avanir Pharmaceuticals. Voelker received summer intern support from Pfizer and the AMCP Foundation during the time of this study. The remaining authors have nothing to disclose.

All authors contributed to study concept and design and to the revision of the manuscript. Bellows, Olsen, and Voelker collected the data, assisted by Wander; data interpretation was performed primarily by Bellows, along with Olsen and Voelker and assisted by Wander. The manuscript was primarily written by Bellows, along with the other authors. 


\section{Antihyperlipidemic Medication Treatment Patterns and Statin Adherence Among Patients with ASCVD in a Managed Care Plan After Release of the 2013 ACC/AHA Guideline on the Treatment of Blood Cholesterol}

\section{REFERENCES}

1. Stone NJ, Robinson JG, Lichtenstein AH, et al. 2013 ACC/AHA guideline on the treatment of blood cholesterol to reduce atherosclerotic cardiovascular risk in adults: a report of the American College of Cardiology/American Heart Association Task Force on Practice Guidelines. J Am Coll Cardiol. 2014;63(25 Pt B):2889-934.

2. National Cholesterol Education Program (NCEP) Expert Panel on Detection, Evaluation, and Treatment of High Blood Cholesterol in Adults (Adult Treatment Panel III). Third Report of the National Cholesterol Education Program (NCEP) Expert Panel on Detection, Evaluation, and Treatment of High Blood Cholesterol in Adults (Adult Treatment Panel III) final report. Circulation. 2002;106(25):3143-421.

3. Pencina MJ, Navar-Boggan AM, D'Agostino RB Sr, et al. Application of new cholesterol guidelines to a population-based sample. N Engl J Med. 2014;370(15):1422-31.

4. Choudhry NK, Avorn J, Glynn RJ, et al. Full coverage for preventive medications after myocardial infarction. N Engl J Med. 2011;365(22):2088-97.

5. Allonen J, Nieminen MS, Lokki M, et al. Mortality rate increases steeply with nonadherence to statin therapy in patients with acute coronary syndrome. Clin Cardiol. 2012;35(11):E22-27.

6. Ho PM, Magid DJ, Shetterly SM, et al. Medication nonadherence is associated with a broad range of adverse outcomes in patients with coronary artery disease. Am Heart J. 2008;155(4):772-79.

7. Jackevicius CA, Mamdani M, Tu JV. Adherence with statin therapy in elderly patients with and without acute coronary syndromes. JAMA. 2002;288(4):462-67.
8. Bouchard MH, Dragomir A, Blais L, Berard A, Pilon D, Perreault S. Impact of adherence to statins on coronary artery disease in primary prevention. Br J Clin Pharmacol. 2007;63(6):698-708

9. Benner JS, Glynn RJ, Mogun H, Neumann PJ, Weinstein MC, Avorn J. Long-term persistence in use of statin therapy in elderly patients. JAMA. 2002;288(4):455-61.

10. Compliance and adverse event withdrawal: their impact on the West of Scotland Coronary Prevention Study. Eur Heart J. 1997;18(11):1718-24.

11. Kumbhani DJ, Steg PG, Cannon CP, et al. Adherence to secondary prevention medications and four-year outcomes in outpatients with atherosclerosis. Am J Med. 2013;126(8):693-700.el.

12. Naderi SH, Bestwick JP, Wald DS. Adherence to drugs that prevent cardiovascular disease: meta-analysis on 376,162 patients. Am J Med. 2012;125(9):882-87.el.

13. Perreault S, Dragomir A, Blais L, et al. Impact of better adherence to statin agents in the primary prevention of coronary artery disease. Eur J Clin Pharmacol. 2009;65(10):1013-24.

14. Budtz-Jørgensen E, Keiding N, Grandjean P, Weihe P. Confounder selection in environmental epidemiology: assessment of health effects of prenatal mercury exposure. Ann Epidemiol. 2007;17(1):27-35.

15. Bruckert E, Hayem G, Dejager S, Yau C, Begaud B. Mild to moderate muscular symptoms with high-dosage statin therapy in hyperlipidemic patients: the PRIMO study. Cardiovasc Drugs Ther. 2005;19(6):403-14.

16. Cohen JD, Brinton EA, Ito MK, Jacobson TA. Understanding Statin Use in America and Gaps in Patient Education (USAGE): an internet-based survey of 10,138 current and former statin users. J Clin Lipidol. 2012;6(3):208-15. 


\section{Antihyperlipidemic Medication Treatment Patterns and Statin Adherence Among Patients with ASCVD in a Managed Care Plan After Release of the 2013 ACC/AHA Guideline on the Treatment of Blood Cholesterol}

\section{APPENDIX A List of ICD-9-CM Codes Used}

Condition

Atherosclerotic cardiovascular disease

Heart disease

Cerebrovascular disease

Atherosclerosis

Peripheral vascular disease

Congenital heart anomaly

Complications after cardiac procedures

Post-procedural status

Aortic aneurysm

Cardiovascular- or lipid-related outpatient visits

Heart disease

Cerebrovascular disease

\begin{tabular}{l}
\hline Atherosclerosis \\
\hline Peripheral vascular disease \\
\hline Congenital heart anomaly \\
\hline Complications after cardiac procedures
\end{tabular}

Postprocedural status

\section{Hyperlipidemia \\ Aortic aneurysm \\ Comorbidities}

\begin{tabular}{l|l}
\hline Anxiety & $300.0^{*}$ \\
\hline \multirow{2}{*}{ Depression } & $296.2^{*}$ \\
\cline { 2 - 2 } & $296.3^{*}$ \\
\cline { 2 - 2 } & 311 \\
\hline
\end{tabular}

\section{Code}

412

413.*

414.**

415.**

416.*

417.*

427 .** $^{*}$

428 .**

$429 . * *$

430

431

$432 *$

433.*

434.*

435.*

436

437.*

438 * $^{*}$

$440 . * *$

$443 . * *$

746.85

996.0*

996.1

996.61

996.71

996.72

996.74

V45.0*

$\mathrm{V} 45.81$

272.*

$441 .^{* *}$
Description

\begin{tabular}{|l|l|}
\hline $410 . * *$ & Acute myocardial infarction \\
\hline $411 .^{* *}$ & Other acute and subacute forms of ischemic heart disease \\
\hline 412 & Old myocardial infarction \\
\hline $413 . *$ & Angina pectoris \\
\hline $414 .^{* *}$ & Other forms of chronic ischemic heart disease \\
\hline 429.2 & Cardiovascular disease, unspecified \\
\hline 437.1 & Other generalized ischemic cerebrovascular disease \\
\hline $440 . * *$ & Atherosclerosis \\
\hline $443 . * *$ & Other peripheral vascular disease \\
\hline 746.85 & Coronary artery anomaly \\
\hline 996.03 & Mechanical complication due to coronary bypass graft \\
\hline V45.81 & Aortocoronary bypass status \\
\hline $441 . * *$ & Aortic aneurysm and dissection \\
\hline
\end{tabular}

411.** $\quad$ Other acute and subacute forms of ischemic heart disease

Old myocardial infarction

Angina pectoris

Other forms of chronic ischemic heart disease

Acute pulmonary heart disease

Chronic pulmonary heart disease

Other diseases of pulmonary circulation

Cardiac dysrhythmias

Heart failure

Ill-defined descriptions and complications of heart disease

Subarachnoid hemorrhage

Intracerebral hemorrhage

Other and unspecified intracranial hemorrhage

Occlusion and stenosis of precerebral arteries

Occlusion of cerebral arteries

Transient cerebral ischemia

Acute, but ill-defined, cerebrovascular disease

Other and ill-defined cerebrovascular disease

Late effects of cerebrovascular disease

Atherosclerosis

Other peripheral vascular disease

Coronary artery anomaly

Mechanical complication of cardiac device implant and graft

Mechanical complication of other vascular device, implant, and graft

Infection and inflammatory reaction due to cardiac device, implant, and graft

Other complications due to heart valve prosthesis

Other complications due to other cardiac device, implant, and graft

Other complications due to other vascular device, implant, and graft

Cardiac device in situ

Aortocoronary bypass status

Disorders of lipoid metabolism

Aortic aneurysm and dissection

300.0* $\quad$ Anxiety states

Major depressive disorder, single occurrence

Major depressive disorder, recurring

Depressive disorder not otherwise specified 
Antihyperlipidemic Medication Treatment Patterns and Statin Adherence Among Patients with ASCVD in a Managed Care Plan After Release of the 2013 ACC/AHA Guideline on the Treatment of Blood Cholesterol

APPENDIX A List of ICD-9-CM Codes Used (continued)

\begin{tabular}{|c|c|c|}
\hline Condition & Code & Description \\
\hline \multicolumn{3}{|l|}{ Comorbidities } \\
\hline \multirow[t]{7}{*}{ Bipolar disorder } & $296.0 *$ & Bipolar I disorder, single manic episode \\
\hline & $296.4 *$ & Bipolar I disorder, most recent episode (or current) manic \\
\hline & $296.5^{*}$ & Bipolar I disorder, most recent episode (or current) depressed \\
\hline & $296.6^{*}$ & Bipolar I disorder, most recent episode (or current) mixed \\
\hline & $296.7^{*}$ & Bipolar I disorder, most recent episode (or current) unspecified \\
\hline & 296.80 & Bipolar disorder, unspecified \\
\hline & 296.89 & Other bipolar disorders \\
\hline \multirow[t]{3}{*}{ Psychotic disorders } & $295 . * *$ & Schizophrenia disorders \\
\hline & $297 . * *$ & Delusional disorders \\
\hline & $298 .^{* *}$ & Other non-organic psychoses \\
\hline Hyperlipidemia & $272 .^{*}$ & Disorders of lipoid metabolism \\
\hline \multirow[t]{5}{*}{ Hypertension } & $401 .^{*}$ & Essential hypertension \\
\hline & $402 . * *$ & Hypertensive heart disease \\
\hline & 403.** & Hypertensive chronic kidney disease \\
\hline & $404 .^{* *}$ & Hypertensive heart and chronic kidney disease \\
\hline & $405 . * *$ & Secondary hypertension \\
\hline Diabetes & $250 . * *$ & Diabetes mellitus \\
\hline Overweight & 278.02 & Overweight \\
\hline \multirow[t]{2}{*}{ Obesity } & 278.00 & Obesity, unspecified \\
\hline & 278.01 & Morbid obesity \\
\hline
\end{tabular}


Antihyperlipidemic Medication Treatment Patterns and Statin Adherence Among Patients with ASCVD in a Managed Care Plan After Release of the 2013 ACC/AHA Guideline on the Treatment of Blood Cholesterol

APPENDIX B Baseline Characteristics of Patients in the Antihyperlipidemic Medication Treatment Patterns Cohort After Post Hoc Matching on Pre-index Antihyperlipidemic Class

\begin{tabular}{|c|c|c|c|c|c|c|c|}
\hline & \multicolumn{2}{|c|}{ Overall $(\mathrm{N}=1,526)$} & \multicolumn{2}{|c|}{ Guideline $(n=763)$} & \multicolumn{2}{|c|}{ Historical $(\mathrm{n}=763)$} & \multirow[b]{2}{*}{$P$ Value ${ }^{a}$} \\
\hline & $\mathrm{n}$ & $\%$ & $\mathrm{n}$ & $\%$ & $\mathrm{n}$ & $\%$ & \\
\hline \multicolumn{8}{|l|}{ Demographics } \\
\hline Age, mean [SD] & 59.5 & [8.1] & 60.5 & [8.6] & 58.6 & {$[7.5]$} & $<0.001$ \\
\hline Male & 1,110 & 72.7 & 543 & 71.2 & 567 & 74.3 & 0.187 \\
\hline \multicolumn{8}{|l|}{ Clinical characteristics } \\
\hline Hyperlipidemia & 1,232 & 80.7 & 610 & 79.9 & 622 & 81.5 & 0.454 \\
\hline Hypertension & 1,124 & 73.7 & 560 & 73.4 & 564 & 73.9 & 0.860 \\
\hline Diabetes & 260 & 17.0 & 130 & 17.0 & 130 & 17.0 & 1.000 \\
\hline Overweight & 13 & 0.9 & 5 & 0.7 & 8 & 1.0 & 0.579 \\
\hline Obesity & 196 & 12.8 & 77 & 10.1 & 119 & 15.6 & 0.002 \\
\hline Anxiety & 19 & 1.2 & 11 & 1.4 & 8 & 1.0 & 0.646 \\
\hline Depression & 42 & 2.8 & 22 & 2.9 & 20 & 2.6 & 0.877 \\
\hline Bipolar & 8 & 0.5 & 7 & 0.9 & 1 & 0.1 & 0.077 \\
\hline Psychotic disorder & 3 & 0.2 & 1 & 0.1 & 2 & 0.3 & 1.000 \\
\hline \multicolumn{8}{|l|}{ Pre-index antihyperlipidemic medication use } \\
\hline \multicolumn{8}{|l|}{ Antihyperlipidemic medication classes } \\
\hline None & 370 & 24.2 & 185 & 24.2 & 185 & 24.2 & \multirow[t]{5}{*}{1.000} \\
\hline Nonstatin medication & 160 & 10.5 & 80 & 10.5 & 80 & 10.5 & \\
\hline Low-intensity statin & 20 & 1.3 & 10 & 1.3 & 10 & 1.3 & \\
\hline Moderate-intensity statin & 728 & 47.7 & 364 & 47.7 & 364 & 47.7 & \\
\hline High-intensity statin & 248 & 16.3 & 124 & 16.3 & 124 & 16.3 & \\
\hline Antihyperlipidemic copay, mean $[S D]^{b}$ & $\$ 22$ & [\$26] & $\$ 19$ & [\$26] & $\$ 24$ & [\$26] & $<0.001$ \\
\hline Number of antihyperlipidemic fills, mean $[S D]^{b}$ & 7.6 & [5.3] & 6.6 & {$[4.3]$} & 8.5 & {$[6.1]$} & $<0.001$ \\
\hline \multicolumn{8}{|c|}{$\begin{array}{l}\text { aContinuous variables compared between guideline and historical groups using paired t-tests, and categorical variables compared using McNemar's test or Maxwell-Stuart } \\
\text { test as appropriate. } \\
\text { bAmong patients with at least } 1 \text { antihyperlipidemic fill. } \\
\text { SD=standard deviation. }\end{array}$} \\
\hline
\end{tabular}

